\title{
All-trans retinoic acid inhibits tumor growth of human osteosarcoma by activating Smad signaling-induced osteogenic differentiation
}

\author{
QIU-JUN YANG ${ }^{1,2}$, LONG-YANG ZHOU ${ }^{1,2}$, YU-QIN MU $^{1,2}$, QI-XIN ZHOU ${ }^{2}$, JIN-YONG LUO $^{1}$, LANG CHENG $^{3}$, \\ ZHONG-LIANG DENG ${ }^{3}$, TONG-CHUAN HE ${ }^{1,4}$, REX C. HAYDON ${ }^{4}$ and BAI-CHENG HE ${ }^{1,2}$ \\ ${ }^{1}$ Key Laboratory of Diagnostic Medicine designated by the Chinese Ministry of Education, Departments of ${ }^{2}$ Pharmacology, \\ and ${ }^{3}$ Orthopedic, The Second Affilited Hospital, Chongqing Medical University, Chongqing, P.R. China; ${ }^{4}$ Molecular \\ Oncology Laboratory, Department of Surgery, The University of Chicago Medical Center, Chicago, IL, USA
}

Received December 10, 2011; Accepted February 23, 2012

DOI: 10.3892/ijo.2012.1426

\begin{abstract}
Osteosarcoma (OS) is one of the most common malignant bone tumors. Despite the advancement of diagnosis and treatment for OS, the prognosis remains poor. We investigated the proliferation inhibitory effect of all-trans retinoic acid (ATRA) for human OS and the possible mechanism underlying this effect. We examined the proliferation inhibition and apoptosis-inducing effects of ATRA in 143B OS cells. We validated this effect by exogenously expressing the retinoic acid receptor alpha (RAR $\alpha$ ) in 143B OS cells and injecting the cells into nude mice. We explored the possible mechanism for the proliferation inhibitory effect of ATRA on OS cells and multipotential progenitor cells by detecting osteogenic markers. We demonstrated that the endogenous retinoic acid receptor and retinoid $\mathrm{X}$ receptor are all detectable in the commercially available OS cell lines and in primary osteosarcoma cells. ATRA inhibits the proliferation of OS cells in a concentration-dependent manner, as well as induces apoptosis in 143B OS cells. The exogenous expression of RAR $\alpha$ inhibits the tumor growth and cell proliferation in vivo. The alkaline phosphatase activity, protein levels of osteopontin (OPN) and osteocalcin (OCN) are all promoted by ATRA in OS cells and mouse embryonic fibroblasts (MEFs), at least by activating the Smad signaling pathway. Collectively, our results strongly indicate that ATRA can inhibit the tumor growth of OS by promoting osteogenic differentiation in OS cells, which is mediated in part by activating Smad signaling. Therefore, combination of ATRA with other current chemotherapy agents may be a promising therapy strategy for OS treatment.
\end{abstract}

Correspondence to: Dr Bai-Cheng He, Department of Diagnostic Medicine, Pharmacy School, Chongqing Medical University, No. 1 Yixueyuan Road, Yuzhong District, Chongqing 400016, P.R. China E-mail: hebaicheng99@yahoo.com

Key words: all-trans retinoic acid, osteosarcoma, proliferation inhibition, osteogenic differentiation, Smad signaling

\section{Introduction}

Osteosarcoma (OS) is one of the most common non-hematologic malignant bone cancers, mainly occurring in children and adolescents. Although chemotherapy and other regiments have been introduced for OS management, few patients with OS can achieve long-term disease-free status. This may be due to the high propensity for pulmonary metastasis and relapse of OS, as well as the serious adverse effects of current chemotherapy agents (1). To date, the molecular pathogenesis of OS is not fully understood. Although some genetic or acquired conditions can increase the risk for OS incidence, such as patients with hereditary retinoblastoma have a high risk for OS $(2,3)$, a variety of genetic alterations resulting in inactivating tumor suppressor genes and over-expression of oncogenes have been reported in OS (4-7), it is still unclear how much these contribute to the development of OS.

Increasing evidence suggests that defects in osteogenic differentiation may lead to OS development $(7,8)$. Osteogenic differentiation is a multi-step process that needs to be well orchestrated in the progenitor cells (9-11). If the progenitor cell deposits defects at the early stage of osteogenic differentiation, it may lead to the development of less differentiated and more malignant OS tumors. On the contrary, OS tumors may be lower grade if they are caused by defects at the later stage in osteogenic differentiation.

In order to improve the prognosis of OS, it is necessary to explore some new treatment strategies, in which differentiation therapy is one of the promising choices (12-14). A few agents have been used as differentiation promoter to treat certain cancers (15-18). ATRA is one of them and has been used to treat acute promyelocytic leukemia (APL) successfully (19).

Retinoic acids (RAs) are derivates of vitamin A and play an important role in embryonic development and function maintenance of vital organs in adult. RAs regulate differentiation and metabolism by activating the RA receptor (RAR) that bind to the retinoid $\mathrm{X}$ receptor (RXR) as a heterodimer. The dimer binds to a regulatory DNA element (retinoic acid response elements, RAREs) and regulates the transcription level of downstream target genes. RAR agonists, such as ATRA, have been 
shown to promote the terminal differentiation of progenitor cells and cancer cells $(15,20)$. ATRA can activate the retinoic acid signaling pathway and cross-talk with other pathways, such as mitogen activated protein kinase (MAPKs) (21), NF- $\mathrm{KB}(22)$ TGF- $\beta(23,24)$ and Notch $(25)$.

Thus, we investigated the anti-proliferation effect of ATRA in human OS cells. We found that the endogenous RAR and RXR present in the commercial available OS cell lines and the primary OS cells as previously described (26). ATRA can inhibit proliferation and induce apoptosis in OS cells. In the in vivo study, we found that exogenous expression of RAR $\alpha$ inhibits OS tumor growth. Further analysis indicates that ATRA can promote osteogenic differentiation in OS cells and MEFs, as well as increase the phosphorylation of Smad1/5/8. Taken together, our data suggest that ATRA can inhibit the OS cell proliferation, this function may be mediated at least by promoting OS cells to undergo osteogenic differentiation through activating Smad signaling.

\section{Materials and methods}

Cell culture and reagents. OS cell line 143B was purchased from American Type Culture Collection, and ATRA from Bimol. Antibodies were from Santa Cruz Biotechnology. All other reagents were purchased form Sigma-Aldrich or Fisher Scientific, unless otherwise indicated. Cells were maintained in the Dulbecco's modified Eagle's medium (DMEM) with $10 \%$ fetal bovine serum (FBS), $100 \mathrm{U} / \mathrm{ml}$ of penicillin and 100 $\mu \mathrm{g} / \mathrm{ml}$ of streptomycin at $37^{\circ} \mathrm{C}$ in $5 \% \mathrm{CO}_{2}$.

Recombinant adenovirus expressing red fluorescent protein and $R A R \alpha$. Recombinant adenoviruses expressing red fluorescent protein (RFP, Ad-RFP) and RAR $\alpha$, (Ad-RAR $\alpha$ ) were constructed with AdEasy system as reported (27). Ad-RFP was used as a mock control.

Isolation of MEFs. MEFs were isolated from post coitus day 12.5 mice, as previously described (28). Each embryo was dissected into $10 \mathrm{ml}$ sterile PBS, voided of its internal organs, and sheared through an 18-gauge syringe in the presence of $1 \mathrm{ml} 0.25 \%$ trypsin and $1 \mathrm{mM}$ EDTA. After 15-min incubation with gentle shaking at $37^{\circ} \mathrm{C}$, DMEM with $10 \%$ FBS was added to inactivate trypsin. The cells were plated on $100-\mathrm{mm}$ dishes and incubated for $24 \mathrm{~h}$ at $37^{\circ} \mathrm{C}$. Adherent cells were used as MEFs. Aliquots were kept in a liquid nitrogen tank. All MEFs used in this study were less than five passages.

Protein harvest and Western blot assay. Cell lysates were denatured and loaded on to SDS-PAGE gel. After electrophoresis, proteins were transferred to polyvinylidene fluoride (PVDF) membrane, and then was blocked with 5\% non-fat milk at room temperature for $1 \mathrm{~h}$ and probed with first antibody for $2 \mathrm{~h}$, followed by 25 -min incubation with second antibody conjugated with horseradish peroxidase, then developed with SuperSignal West Pico Chemiluminescent Substrate kit. Images were taken with Bio-Rad imaging system.

Cell proliferation assay. Sub-confluent cells were seeded in 24-well plates and treated with different concentrations of ATRA or dimethyl sulfoxide (DMSO) as control. At the scheduled time- points, cells were stained with crystal violet to visualize the cell viability, followed by quantitative analysis with Image J (1.42q).

Apoptosis staining. Sub-confluent cells were seeded in 6-well plates and treated with $80 \mu \mathrm{M}$ ATRA or DMSO. Cells were collected $48 \mathrm{~h}$ later, followed by staining for apoptosis detection with Vybrant Apoptosis Assay Kit (V-23201, Invitrogen, USA). For fluorescence activated cell sorting (FACS) assay, cells were collected and subjected to staining with propidium iodide (PI) and Annex-V FITC (no. KGA104, KeyGen Biotech, China) for apoptosis.

Establishment of the stable OS cell line expressing firefly luciferase. Stably expressing firefly luciferase 143B cell line (143B-Luc) was generated by using a retroviral vector and 143B cells as described (29).

Orthotopic OS tumor animal model establishment. All animal experiments follow the guideline of Institutional Animal Care and Use Committee of The University of Chicago (IACUC). Athymic mice (female, 4-6-week old, 5/group) were obtained from Harlan Sprague Dawley (USA).

143B-luc cells were infected with Ad-RAR $\alpha$ or Ad-RFP adenovirus. Cells were collected and resuspended in cold $\left(4^{\circ} \mathrm{C}\right)$ phosphate buffer solution (PBS) to a final density of $2 \times 10^{7}$ cell/ $\mathrm{ml}$. Cells in $50 \mu \mathrm{l}$ of cold PBS were injected into the proximal tibia of athymic mice (female, 4-6-week old). Animals were sacrificed 5 weeks after injection. The tumor samples were retrieved for histochemical assay.

In vivo bioluminescence imaging. Animals were anesthetized with isoflurane attached to a nose-cone mask within Xenogen IVIS200 imaging system. Animals were injected with D-Luciferin sodium salt (Gold Biotechnology) at $100 \mathrm{mg} / \mathrm{kg}$ in $0.1 \mathrm{ml}$ sterile PBS. The pseudoimage were obtained by superimposing the emitted light over the gray scale photographs of the animals. Quantitative analysis was done with the Xenogen Living Image Software (250.1).

Histologic and immunohistochemical staining. Animals were sacrificed at the end of test, the retrieved tumor samples were fixed in $10 \%$ formalin (decalcified if necessary) and embedded in paraffin. Sections of the embedded specimens were stained with hematoxylin and eosin (H\&E). For proliferating cell nuclear antigen (PCNA) immunohistochemical staining, sections were deparaffinized and then rehydrated in graduated fashion. The deparaffinized samples were subjected to antigen retrieval and fixation. Slides were blocked and probed with PCNA antibody. PCNA was visualized by 3,3'-diaminobenzidine (DAB) staining, mouse IgG was run as a negative control.

Alkaline phosphatase activity assay. Alkaline phosphatase (ALP) activities were detected by a modified protocol, using p-nitrophenyl phosphate as a substrate, as described $(30,31)$. Briefly, sub-confluent OS cells were seeded in 24-well plates and treated with different concentrations of ATRA. On day 5 and 7 , the cells were lysed and the lysates were centrifuged to discard the precipitation. The supernatant was used for ALP activity test by colorimetric assay. The total protein level was 


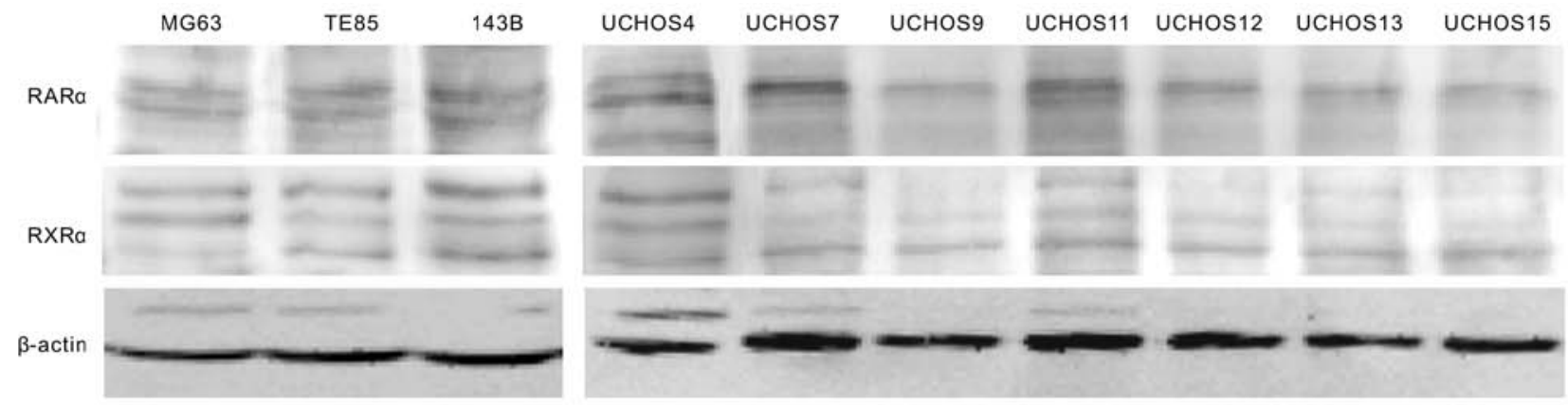

Figure 1. Endogenous expression of RAR $\alpha$ and RXR $\alpha$ in human OS cells and primary OS derived cells. Left three lanes show the basal RAR $\alpha$ and RXR $\alpha$ expression level in the commercially available cell lines, the seven lanes on the right show the basal RAR $\alpha$ and RXR $\alpha$ expression level in primary OS derived cells, $\beta$-actin was used as loading control. The result shows that RAR $\alpha$ and RXR $\alpha$ are all expressed in these OS cells (UCHOS, University of Chicago Human Osteosarcoma).

A

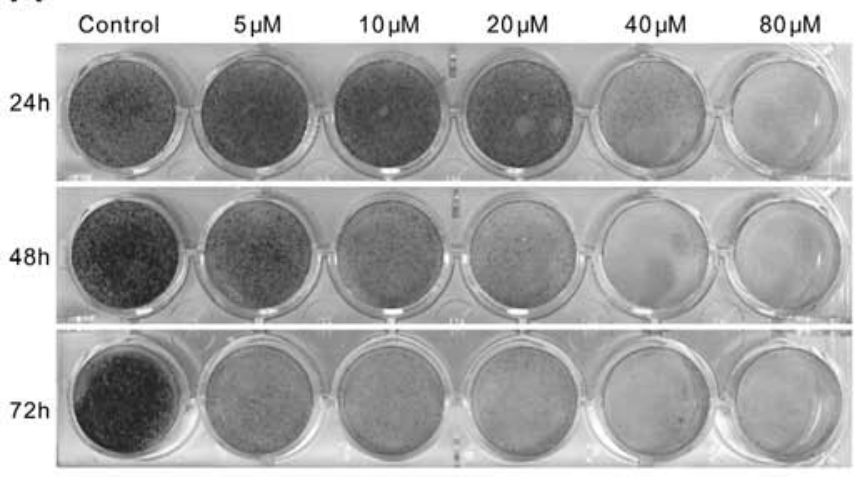

B

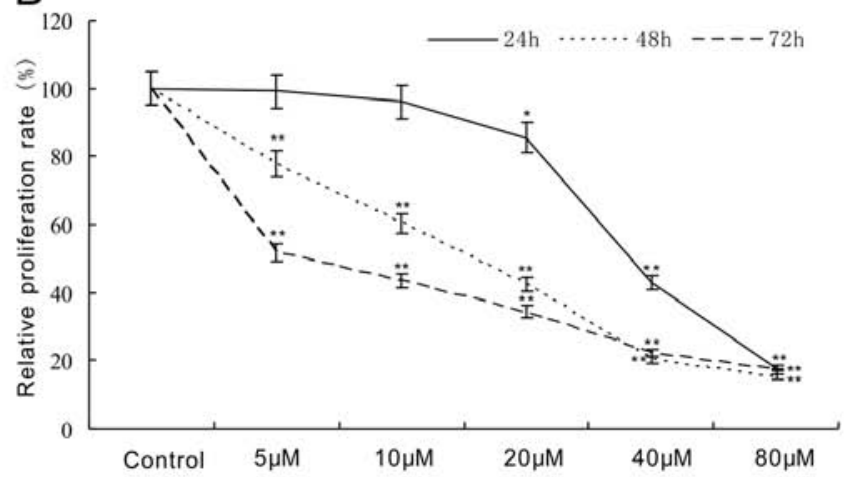

Figure 2. The proliferation inhibitory effect of ATRA on OS cells. (A) The crystal violet staining result in 143B cells. Sub-confluent 143B cells were seeded in 24-well plates and treated with different concentrations of ATRA or DMSO, followed by crystal violet staining at the indicated time-points. (B) The quantitative result of crystal violet staining. The quantification of crystal violet staining results was processed as described in Materials and methods. The assay was done in triplicate. ${ }^{*} \mathrm{p}<0.05$ vs control; ${ }^{* *} \mathrm{p}<0.01$ vs control.

assessed by BCA for normalizing the ALP activity. Each assay was performed in triplicate.

Luciferase reporter assay. Cells were seeded in T25 flask and transfected with $3 \mu \mathrm{g}$ of firefly luciferase reporter plasmids. Twelve hours later, trypsinized cells were seeded to 24-well plates, then treated with ATRA or DMSO. Lysed cells, and the lysates were subjected to luciferase assays using luciferase assay kit (no. TM040, Promega, USA) at 24 h, total protein level was assessed by BCA for normalizing the firefly luciferase activity. Each assay was done in triplicate.

Statistical analysis. Data presented are the results of at least two independent experiments performed in triplicate. Microsoft Excel was employed to calculate the standard deviations. The differences were analyzed using the Student's t-test. A $p<0.05$ was defined as statistically significant.

\section{Results}

Endogenous expression of RAR and RXR in human $O S$ cell lines and primary $O S$ cells. We first detected the endogenous expression level of RAR and RXR in the three commercially available OS cell lines and seven primary human OS cells as previously described (26). The Western blotting results show that RAR $\alpha$ and RXR $\alpha$ were expressed in all of the cells (Fig. 1). We also tested the subtype receptors of RAR and RXR with real-time PCR, each subtype receptor can be detected in the OS cells (data are not shown). Our results suggest that the retinoic acid signaling is available in OS cells.

ATRA inhibits the proliferation of OS cells. To test the proliferation inhibitory effect of ATRA on OS cells, we employed the crystal violet staining. The result shows that the proliferation of 143B cells was inhibited by ATRA with a concentration and time-dependent fashion (Fig. 2A). The staining result was quantified with ImageJ (1.42q) (Fig. 2B). The assay was done in triplicate and the representative result is shown.

ATRA induces apoptosis in OS cells. Next, we tested whether ATRA could induce apoptosis in OS cells. The 143B OS cells were treated with indicated concentrations of ATRA or DMSO for 12 and $24 \mathrm{~h}$. Then, cells were subjected with FACS analysis (Fig. 3A), or were lysed and subjected to Western blot test for detecting caspase-3 protein level (Fig. 3B). The results show that ATRA can induce apoptosis in 143B cells, and the caspase- 3 protein increased at 12 and $24 \mathrm{~h}$ with a concentration-dependent fashion. Cells were also subjected to apoptosis staining after 

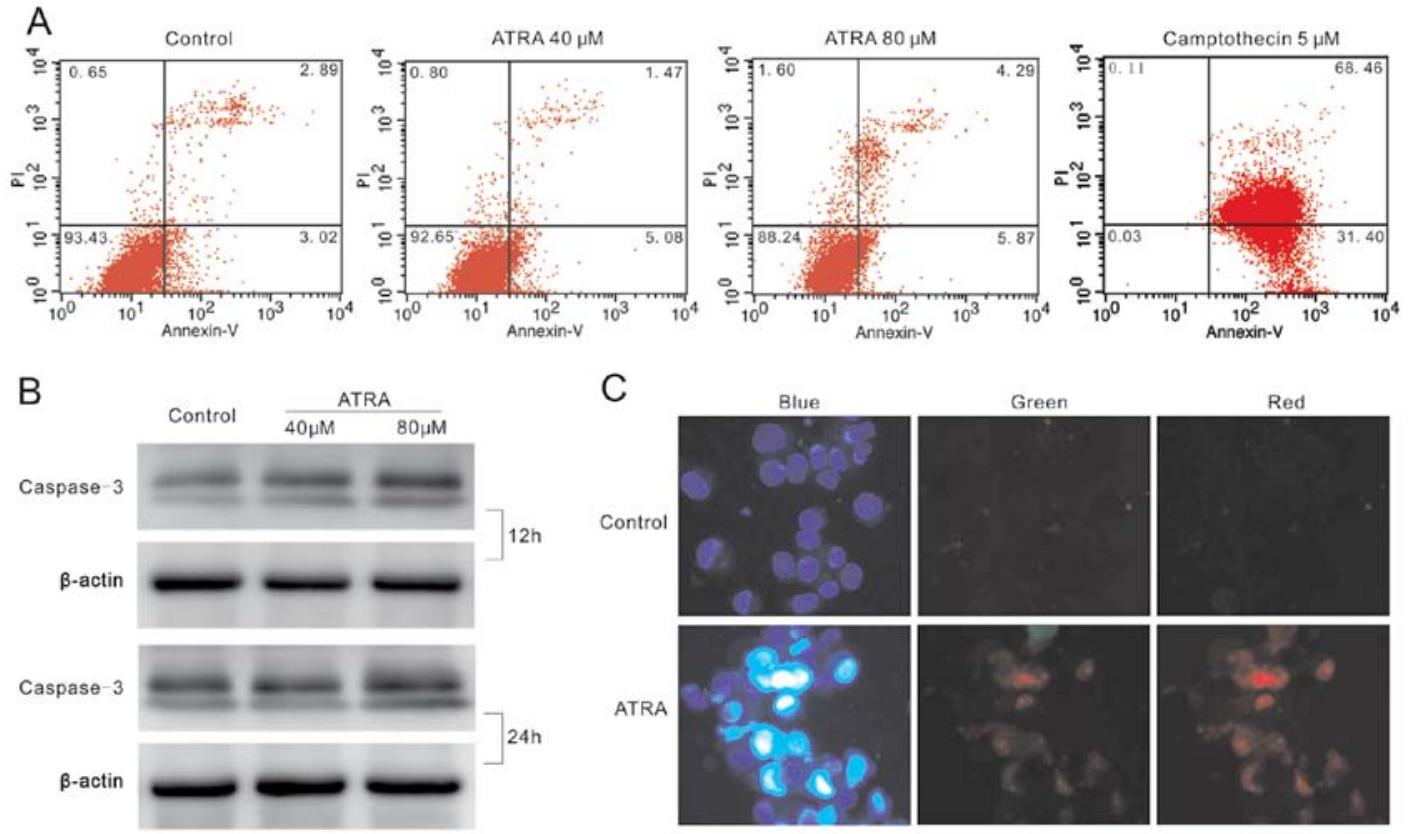

Figure 3. ATRA induces apoptosis in 143B OS cells. (A) The FACS analysis of apoptosis in 143B cells. Sub-confluent 143B cells were seeded in 6-well plates and treated with different concentrations of ATRA or DMSO, tests were processed at $24 \mathrm{~h}$, camptothecin was used as a positive control. (B) Western blot assay for the capase-3 protein level in 143B cells. Cells were seeded in 6-well plates and treated with the indicated concentrations of ATRA or DMSO, the protein sample was harvested at 12 and $24 \mathrm{~h}$ after treatment with ATRA and blotted with caspase-3 antibody. (C) The apoptosis staining results in 143B cells. Cells were seeded in 6-well plates and treated with ATRA $(80 \mu \mathrm{M})$ or DMSO for $24 \mathrm{~h}$, then staining with the kit from Invitrogen. The live cells show only low blue fluorescence (Hoechst 33342), the apoptotic cells show bright blue and green fluorescence (YO-PRO-1), the necrotic cells show red fluorescence (PI), as well as bright blue and green fluorescence. Each assay was performed in triplicate.
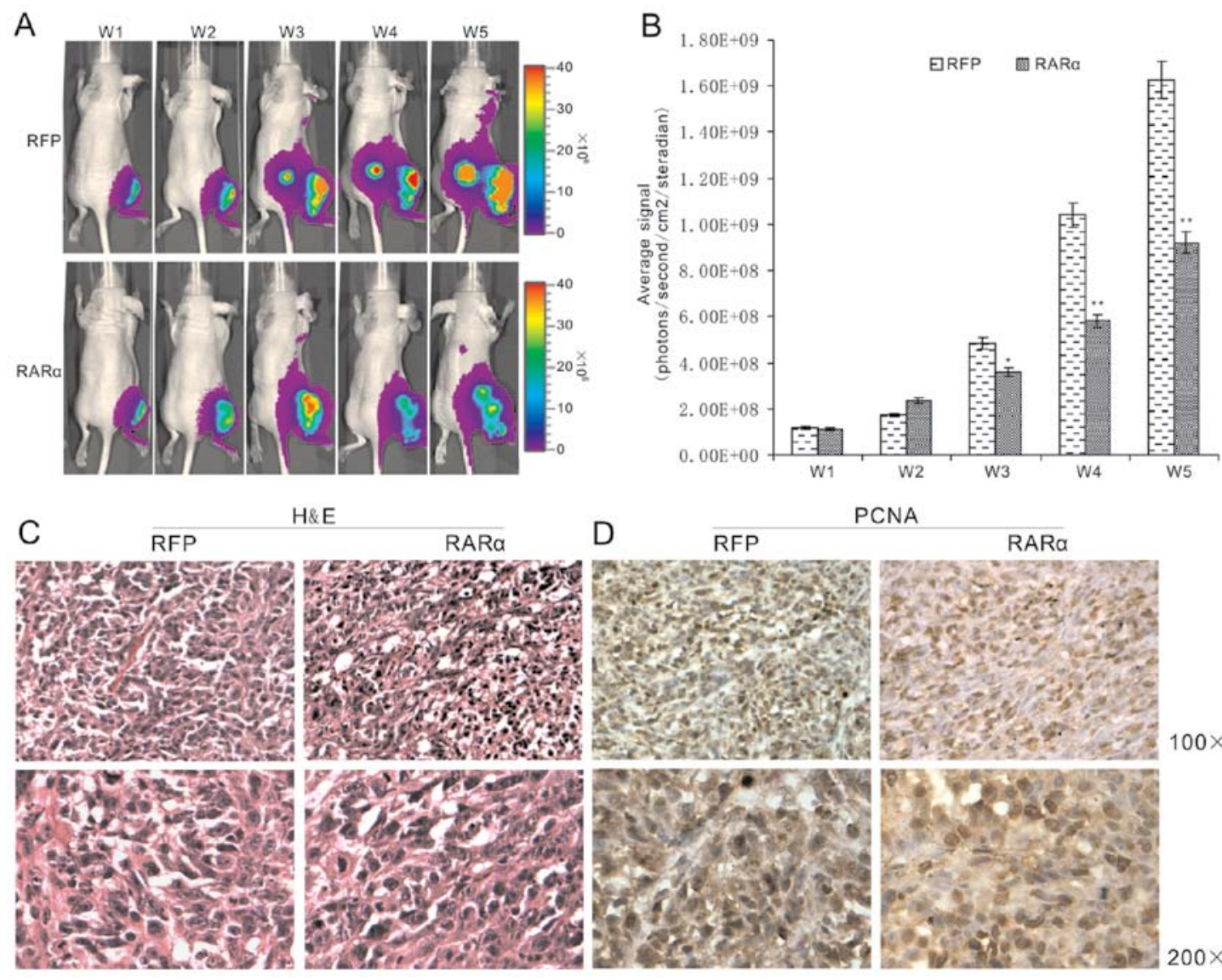

Figure 4. Exogenous expression of RAR $\alpha$ inhibits the OS tumor growth in vivo. To validate the proliferation inhibitory effect the activated retinoic acid signaling in vivo, we infected the firefly luciferase tagged 143B cells (143B-luc) with Ad-RAR $\alpha$ and Ad-RFP (as mock control), then injected the cells to nude mice as reported. Animals were imaged weekly for five weeks with Xenogen imaging system. (A) The representative Xenogen images for the in vivo assay. (B) The quantitative results of Xenogen images with Xenogen living image software (250.1). At the end of imaging, the OS tumors samples were retrieved, fixed and paraffin-embedded. The sections were subjected to H\&E staining (C) and PCNA immunohistochemical staining (D). ${ }^{*}$ p $<0.05$ vs control; * ${ }^{* *}<0.01$ vs control. 

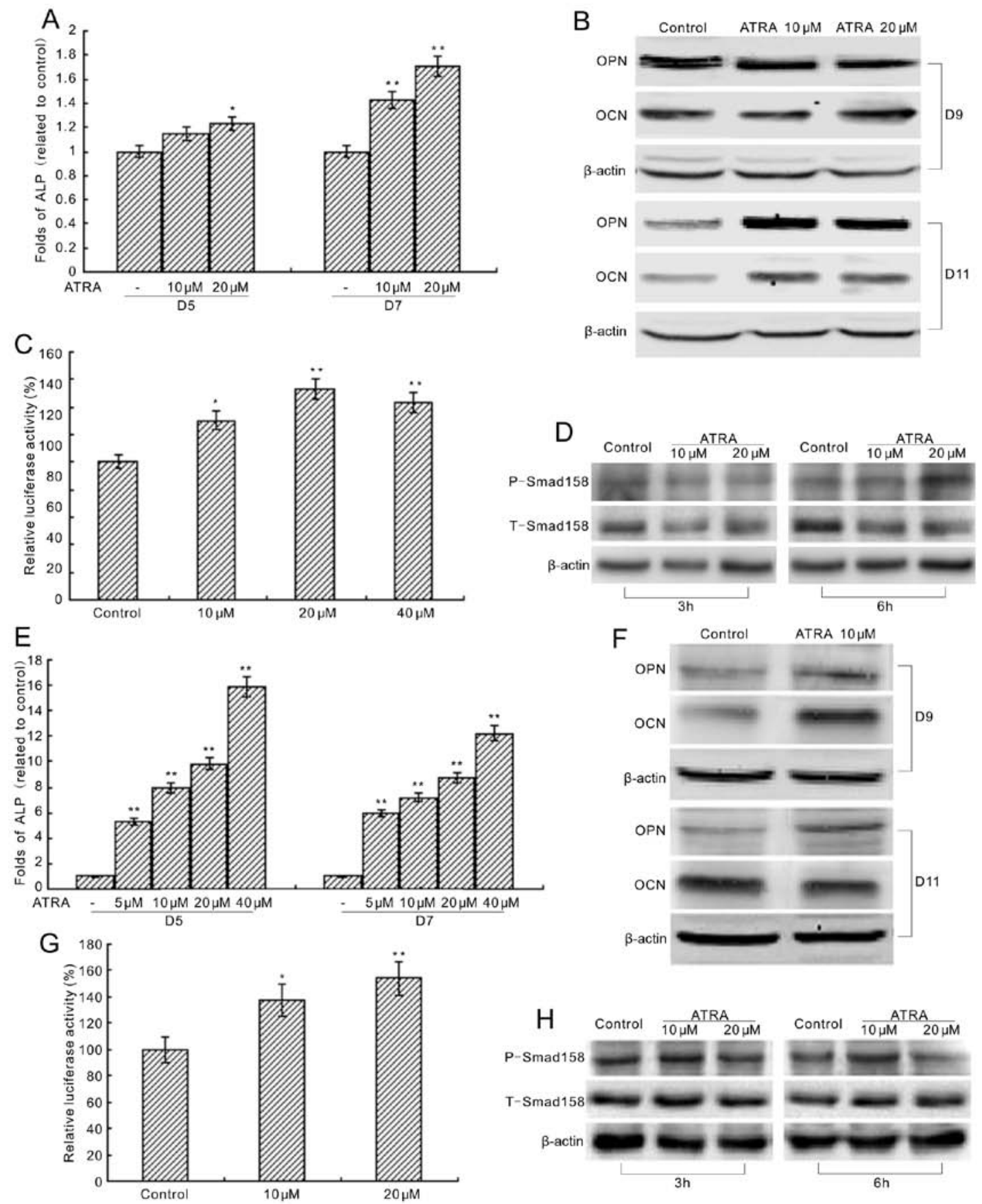

Figure 5. ATRA promotes osteogenic differentiation in 143B OS cells and MEFs by activating Smad signaling. (A) ALP activities assay in OS cells. Cells were seeded in 24-well plates and treated with the indicated concentrations of ATRA or DMSO. The ALP activities were measured on D5 and D7. (B) Western blot assay for OPN and OCN in OS cells. Cells were seeded in 6-well plates and treated with indicated concentrations of ATRA or DMSO, total protein was harvested and blotted with anti-OPN and anti-OCN antibody on D9 and D11. (C) BMPR-Smad binding site reporter assay in OS cells. Cells were seeded in T25 flask and transfected with p12XSBE-Luc plasmids, replated to 24-well plates and treated with different concentration of ATRA or DMSO. The luciferase activity was measured at $24 \mathrm{~h}$ after ATRA treatment. (D) Western blot assay for the phosphorylated Smad1/5/8 in OS cells, sub-confluent cells were seeded in 6-well plates and treated with different concentrations of ATRA for 3 and $6 \mathrm{~h}$. Harvested total protein and blotted with antibody for Smad1/5/8 (T-Smad1/5/8) or phosphorylated Smad1/5/8 (P-Smad1/5/8). $\beta$-actin was used as loading control. Similar results were obtained in MEFs. (E) ALP activity assay in MEFs cells. (F) Western blot assay for OPN and OCN in MEFs cells. (G) BMPR-Smad binding site luciferase reporter assay in MEFs cells. (H) Western blot assay for the phosphorylated Smad1/5/8 in MEFs cells. Each assay was done in triplicate. " $\mathrm{p}<0.05$ vs control; " $\mathrm{p}<0.01$ vs control.

being treated with ATRA for $48 \mathrm{~h}$, the result shows that the apoptosis apparently occurred when treated with $80 \mu \mathrm{M}$ ATRA (Fig. 3C). These data indicate that ATRA can induce apoptosis in OS cells.

Exogenous expression of RAR $\alpha$ inhibits OS tumor growth in vivo. To elucidate whether ARTA can inhibit the proliferation of OS cells in vivo, we tested tumor growth inhibitory effect of ATRA by exogenous expressing RAR $\alpha$ receptor in OS cells. We took advantage of the reported orthotopic xenograft model of OS tumor for this test (32). The luciferase tagged 143B OS cells were infected with Ad-RAR $\alpha$ or Ad-RFP (as mock control), then were injected into athymic nude mice intratibially and Xenogen images were taken weekly (Fig. 4A). The quantitative results 
indicate that the exogenous expression of RAR $\alpha$ can inhibit the OS tumor growth (Fig. 4B). Retrieved samples from the OS tumors were fixed and paraffin-embedded. The sections were subjected to $\mathrm{H} \& \mathrm{E}$ staining and PCNA immunohistochemical staining (Fig. 4C and D). The result validates that ATRA can inhibit the proliferation of OS cells in vivo.

ATRA promotes OS cells to undergo osteogenic differentiation by activating Smad signaling in OS cells. Osteogenic differentiation from osteoprogenitor cells to osteocyte is a well orchestrated process. Related research has shown that OS deposits osteogenic differentiation defects. We tested the possibility of ATRA to promote OS cells to undergo osteogenic differentiation. Our results show that ATRA can increase the ALP activity in 143B OS cells on day 5 and 7 (Fig. 5A), the protein level of OPN and OCN are all increased on day 9 and 11 (Fig. 5B). To further understand the mechanism underlying the osteogenic differentiation induced by ATRA in OS cells, we used bone morphogenic protein (BMP) receptorspecific Smad binding site luciferase reporter (BMPR-Smad, p12XSBE-Luc) (33) and Western blotting to test the activation of Smad signaling. The results show that ATRA can promote the reporter luciferase activity in a concentration-dependent fashion (Fig. 5C), and increase the phosphorylation level of Smad-1/5/8 (Fig. 5D), which is critical for transducing osteogenic signals. In MEFs cells, we obtained similar results. ATRA increased the ALP activities (Fig. 5E), protein expression level of OPN and OCN (Fig. 5F), luciferase activity of p12XSBELuc luciferase reporter (Fig. 5G), and the phosphorylation level of Smad-1/5/8 compared to that of the control group (Fig. 5H). These results suggest that the proliferation inhibitory effect of ATRA on 143B OS cells may result from the promoted osteogenic differentiation, which was at least partly mediated by activating Smad signaling.

\section{Discussion}

OS is a common bone cancer. The current management include chemotherapy, radiotherapy and surgical excise, with marginal improvement of the poor prognosis. It is necessary to find less toxic and more efficacious therapy strategies for OS treatment. Herein, we present a new management strategy for OS with ATRA.

In this study, we demonstrated that the endogenous retinoic acid signaling is available in all tested OS cell lines or OS cells retrieved from primary OS tumors. This evidence suggests that retinoic acid signaling may execute unknown committed functions when activated by their endogenous or exogenous agonists in OS cells. Our results indicate that ATRA can inhibit proliferation and induce apoptosis in OS cells. Exogenous RAR $\alpha$ can inhibit OS tumor growth in an orthotopic animal model in nude mice (32). Further analysis shows that ATRA can promote the osteogenic differentiation in OS cells and MEFs, which may be mediated by activating Smad signaling pathway.

OS is characterized by a bimodal distribution, with the first peak in children and adolescents, the second smaller peak in elderly adults, high incidence of pulmonary metastasis and varying degree of osteoblastic differentiation $(4,5,7,34)$. To date, our knowledge of OS molecular pathogenesis remains rather limited, so it is hard to find specific targets or efficacious treatment to eliminate OS or improve the prognosis of OS. The successful clinical treatment for OS also faces various challenges. 1) The serious adverse effect of the chemotherapy drugs, because most of them are non-specific cytotoxic agents and mostly target the rapid proliferation tumor cells $(10,16,35)$, and 2) the OS harbors propensity to lung metastasis and high recurrence, which produce the majority of OS related mortality (34).

Differentiation therapy may be another promising alternative for certain malignancies. Nuclear receptors, such as RAR and RXR, have been documented as potential targets for cancer treatment (35-37). ATRA, the agonist of RAR, has been used for the management of acute promyelocytic leukemia (APL), one of the most promising mechanisms of ATRA on APL is to promote the immature cells undergo terminal differentiation $(19,38)$. ATRA has also been documented as differentiation and/or apoptosis inducer for the management of certain other cancers, including breast cancer, bladder cancer, Kaposi's sarcoma and neuroblastoma $(19,39)$.

Although the molecular mechanism of OS remains unclear, with the expansion of our knowledge on stem cells and cancer, more and more evidence suggests that OS may be a kind of differentiation disease $(5,8,40)$. Our results show a broad range of ALP activity in OS cell lines or OS cells derivated from the primary OS tumors (data are not shown). Osteoprogenitors differentiate to osteocytes controlled by a variety of signalings (11,30,41-46). Some genetic or epigenetic changes can disturb the osteogenic differentiation process and finally cause the development of OS. This opinion is supported by the following evidence: first, most of OS have the features of undifferentiated osteoblasts $(4,5,7,34)$. Second, some agents can drive OS cells to undergo osteogenic differentiation $(5,35)$. Third, the disturbance of function of RB can attenuate the osteogenic differentiation, because RB is one of the important regulators of Runx-2, a pivotal transcription regulator for osteogenic differentiation $(47,48)$. Fourth, some osteogenic agents fail to drive osteogenic differentiation in most OS cells and promote OS tumor cell growth, such as osteogenic BMPs (5). Finally, in Ewing's sarcoma (EWS), EWS/ETS fusion protein can block the osteogenic and adipogenic differentiation in marrow stromal stem cells (49). The expression of EWS/FLI-1 oncogene can lead to EWS like tumors and EWS/FLI-1 knockdown can drive some ESW cells to differentiate along adipogenic or osteogenic lineages when some appropriate cocktails were used $(50,51)$. Taken together, differentiation therapy, by driving terminal osteogenic differentiation or bypassing the differentiate defects, may be an efficacious regimen or adjuvant combined with the conventional therapy for OS treatment.

Our results indicate that ATRA can inhibit the proliferation of OS. This effect was recapitulated in a well established orthotropic animal model through exogenous expressing RAR $\alpha$. Further analysis suggested that the proliferation inhibitory effect of ATRA on OS cells may be result from promoting the OS cells to undergo osteogenic differentiation. It is possible to establish a novel therapy strategy by combining ATRA with traditional chemotherapy agents for the treatment of OS, which may increase the anti-tumor effects and attenuate the adverse effect of the traditional agents. Future experiments will focus on the possible mechanism underlying the effect of ATRA on OS cells and the possibility of ATRA 
combine with some traditional agents as a new treatment for OS cells.

The role of ATRA in osteogenic differentiation is controversial, some reports indicate that ATRA can inhibit osteogenesis and promote adipogenesis $(46,52,53)$. However, our results in this study and some other results before indicate that ATRA can promote osteogenic differentiation (20). In our opinion ATRA and its analogs are non-specific differentiation promoting agents. It is possible that the outcomes of ATRA treatment may largely depend on the cell types/stages of progenitors used.

Taken together, our study demonstrated that ATRA can inhibit the proliferation of OS cells in vivo and in vitro by promoting OS cells to undergo osteogenic differentiation, which may be mediated by activating Smad signaling.

\section{Acknowledgements}

We thank Dr Di Chen of University of Rochester Medical Center for his kind provision of the p12XSBE-Luc plasmids. This study was supported in part by research grants from Natural Science Foundation of China, Chongqing Science \& Technology Commission of China, the National Basic Research Program of China (NSFC 81071462, CSTC 2011BB5129, 2011CB70790).

\section{References}

1. Geller DS and Gorlick R: Osteosarcoma: a review of diagnosis management, and treatment strategies. Clin Adv Hematol Oncol 8: 705-718, 2010

2. Tateishi U,Hasegawa T, Miyakawa K, Sumi M and Moriyama N: $\mathrm{CT}$ and MRI features of recurrent tumors and second primary neoplasms in pediatric patients with retinoblastoma. AJR Am J Roentgenol 181: 879-884, 2003.

3. Draper GJ, Sanders BM and Kingston JE: Second primary neoplasms in patients with retinoblastoma. Br J Cancer 53: 661-671, 1986.

4. Sandberg AA and Bridge JA: Updates on the cytogenetics and molecular genetics of bone and soft tissue tumors: osteosarcoma and related tumors. Cancer Genet Cytogenet 145: 1-30, 2003.

5. Haydon RC, Luu HH and He TC: Osteosarcoma and osteoblastic differentiation: a new perspective on oncogenesis. Clin Orthop Relat Res 454: 237-246, 2007.

6. Helman LJ and Meltzer P: Mechanisms of sarcoma development. Nat Rev Cancer 3: 685-694, 2003.

7. Tang N, Song WX, Luo J, Haydon RC and He TC: Osteosarcoma development and stem cell differentiation. Clin Orthop Relat Res 466: 2114-2130, 2008

8. Luo X, Chen J, Song WX, et al: Osteogenic BMPs promote tumor growth of human osteosarcomas that harbor differentiation defects. Lab Invest 88: 1264-1277, 2008

9. Aubin JE: Regulation of osteoblast formation and function. Rev Endocr Metab Disord 2: 81-94, 2001.

10. Luu HH, Song WX, Luo X, et al: Distinct roles of bone morphogenetic proteins in osteogenic differentiation of mesenchymal stem cells. J Orthop Res 25: 665-677, 2007.

11. Deng ZL, Sharff KA, Tang N, et al: Regulation of osteogenic differentiation during skeletal development. Front Biosci 13 2001-2021, 2008.

12. Sell S: Stem cell origin of cancer and differentiation therapy. Crit Rev Oncol Hematol 51: 1-28, 2004.

13. Monzillo E and Gronowicz G: New insights on therapeutic touch a discussion of experimental methodology and design that resulted in significant effects on normal human cells and osteosarcoma. Explore (NY) 7: 44-51, 2011.

14. Akiyama T, Dass CR and Choong PF: Novel therapeutic strategy for osteosarcoma targeting osteoclast differentiation, bone-resorbing activity, and apoptosis pathway. Mol Cancer Ther 7: 3461-3469, 2008.

15. Freemantle SJ, Spinella MJ and Dmitrovsky E: Retinoids in cancer therapy and chemoprevention: promise meets resistance. Oncogene 22: 7305-7315, 2003
16. Park BH, Breyer B and He TC: Peroxisome proliferator-activated receptors: roles in tumorigenesis and chemoprevention in human cancer. Curr Opin Oncol 13: 78-83, 2001.

17. Tang XH and Gudas LJ: Retinoids, retinoic acid receptors, and cancer. Annu Rev Pathol 6: 345-364, 2011.

18. Garattini E, Gianni M and Terao M: Retinoid related molecules an emerging class of apoptotic agents with promising therapeutic potential in oncology: pharmacological activity and mechanisms of action. Curr Pharm Des 10: 433-448, 2004.

19. Kamimura T, Miyamoto T, Harada M and Akashi K: Advances in therapies for acute promyelocytic leukemia. Cancer Sci 102: 1929-1937. 2011.

20. Zhang W, Deng ZL, Chen L, et al: Retinoic acids potentiate BMP9induced osteogenic differentiation of mesenchymal progenitor cells. PLoS One 5: e11917, 2010.

21. Karsy M, Albert L, Tobias ME, Murali R and Jhanwar-Uniyal M: All-trans retinoic acid modulates cancer stem cells of glioblastoma multiforme in an MAPK-dependent manner. Anticancer Res 30: 4915-4920, 2010

22. Dutta A, Sen T, Banerji A, Das S and Chatterjee A: Studies on multifunctional effect of all-trans retinoic acid (ATRA) on matrix metalloproteinase-2 (MMP-2) and its regulatory molecules in human breast cancer cells (MCF-7). J Oncol 2009: $627840,2009$.

23. Yu Z and Xing Y: All-trans retinoic acid inhibited chondrogenesis of mouse embryonic palate mesenchymal cells by down-regulation of TGF-beta/Smad signaling. Biochem Biophys Res Commun 340: 929-934, 2006.

24. Yao Z, Chen D, Wang A, et al: Folic acid rescue of ATRA-induced cleft palate by restoring the TGF-beta signal and inhibiting apoptosis. J Oral Pathol Med 40: 433-439, 2011.

25. Ying M, Wang S, Sang Y, et al: Regulation of glioblastoma stem cells by retinoic acid: role for Notch pathway inhibition. Oncogene 30: 3454-3467, 2011

26. Luo X, Sharff KA, Chen J, He TC and Luu HH: S100A6 expression and function in human osteosarcoma. Clin Orthop Relat Res 466: 2060-2070, 2008.

27. He TC, Zhou S, da Costa LT, Yu J, Kinzler KW and Vogelstein B A simplified system for generating recombinant adenoviruses. Proc Natl Acad Sci USA 95: 2509-2514, 1998.

28. Sharff KA, Song WX, Luo X, et al: Heyl basic helix-loop-helix protein plays an important role in mediating BMP9-induced osteogenic differentiation of mesenchymal progenitor cells. J Biol Chem 284: 649-659, 2009

29. Su Y, Luo X, He BC, et al: Establishment and characterization of a new highly metastatic human osteosarcoma cell line. Clin Exp Metastasis 26: 599-610, 2009.

30. Kang Q, Song WX, Luo Q, et al: A comprehensive analysis of the dual roles of BMPs in regulating adipogenic and osteogenic differentiation of mesenchymal progenitor cells. Stem Cells Dev 18: 545-559, 2009.

31. Cheng H, Jiang W, Phillips FM, et al: Osteogenic activity of the fourteen types of human bone morphogenetic proteins (BMPs). J Bone Joint Surg Am 85A: 1544-1552, 2003.

32. Luu HH, Kang Q, Park JK, et al: An orthotopic model of human osteosarcoma growth and spontaneous pulmonary metastasis. Clin Exp Metastasis 22: 319-329, 2005.

33. Zhao M, Qiao M, Oyajobi BO, Mundy GR and Chen D: E3 ubiquitin ligase Smurf1 mediates core-binding factor alpha1/ Runx2 degradation and plays a specific role in osteoblast differentiation. J Biol Chem 278: 27939-27944, 2003.

34. Marina N, Gebhardt M, Teot L and Gorlick R: Biology and therapeutic advances for pediatric osteosarcoma. Oncologist 9: 422-441, 2004.

35. Haydon RC, Zhou L, Feng T, et al: Nuclear receptor agonists as potential differentiation therapy agents for human osteosarcoma. Clin Cancer Res 8: 1288-1294, 2002.

36. Altucci L, Leibowitz MD, Ogilvie KM, de Lera AR and Gronemeyer H: RAR and RXR modulation in cancer and metabolic disease. Nat Rev Drug Discov 6: 793-810, 2007.

37. Krishnan A, Nair SA and Pillai MR: Biology of PPAR gamma in cancer: a critical review on existing lacunae. Curr Mol Med 7: 532-540, 2007.

38. Kambhampati S, Verma A, Li Y, Parmar S, Sassano A and Platanias LC: Signalling pathways activated by all-trans-retinoic acid in acute promyelocytic leukemia cells. Leuk Lymphoma 45: 2175-2185, 2004.

39. Park JH and Tallman MS: Managing acute promyelocytic leukemia without conventional chemotherapy: is it possible? Expert Rev Hematol 4: 427-436, 2011. 
40. Thomas D and Kansara M: Epigenetic modifications in osteogenic differentiation and transformation. J Cell Biochem 98: 757-769, 2006.

41. Tang N, Song WX, Luo J, et al: BMP-9-induced osteogenic differentiation of mesenchymal progenitors requires functional canonical Wnt/beta-catenin signalling. J Cell Mol Med 13: 2448-2464, 2009.

42. Hong JH, Hwang ES, McManus MT, et al: TAZ, a transcriptional modulator of mesenchymal stem cell differentiation. Science 309: 1074-1078, 2005.

43. Karsenty G: Role of Cbfa1 in osteoblast differentiation and function. Semin Cell Dev Biol 11: 343-346, 2000.

44. Nakashima K, Zhou X, Kunkel G, et al: The novel zinc fingercontaining transcription factor osterix is required for osteoblast differentiation and bone formation. Cell 108: 17-29, 2002.

45. Luo J, Tang M, Huang J, et al: TGFbeta/BMP type I receptors ALK1 and ALK2 are essential for BMP9-induced osteogenic signaling in mesenchymal stem cells. J Biol Chem 285: 2958829598, 2010.

46. Wan DC, Shi YY, Nacamuli RP, Quarto N, Lyons KM and Longaker MT: Osteogenic differentiation of mouse adipose-derived adult stromal cells requires retinoic acid and bone morphogenetic protein receptor type IB signaling. Proc Natl Acad Sci USA 103 12335-12340, 2006.
47. Thomas DM, Johnson SA, Sims NA, et al: Terminal osteoblast differentiation, mediated by runx 2 and $\mathrm{p} 27^{\mathrm{KIP} 1}$, is disrupted in osteosarcoma. J Cell Biol 167: 925-934, 2004.

48. Van Deursen JM: Rb loss causes cancer by driving mitosis mad. Cancer Cell 11: 1-3, 2007.

49. Tucker MA, D'Angio GJ, Boice JD Jr, et al: Bone sarcomas linked to radiotherapy and chemotherapy in children. N Engl J Med 317: 588-593, 1987

50. Castillero-Trejo Y, Eliazer S, Xiang L, Richardson JA and Ilaria RL Jr: Expression of the EWS/FLI-1 oncogene in murine primary bone-derived cells results in EWS/FLI-1-dependent, Ewing sarcoma-like tumors. Cancer Res 65: 8698-8705, 2005.

51. Tirode F, Laud-Duval K, Prieur A, Delorme B, Charbord P and Delattre O: Mesenchymal stem cell features of Ewing tumors. Cancer Cell 11: 421-429, 2007.

52. Kneissel M, Studer A, Cortesi R and Susa M: Retinoid-induced bone thinning is caused by subperiosteal osteoclast activity in adult rodents. Bone 36: 202-214, 2005.

53. Mercader J, Palou A and Bonet ML: Induction of uncoupling protein-1 in mouse embryonic fibroblast-derived adipocytes by retinoic acid. Obesity 18: 655-662, 2010. 